\title{
SNAP: Spreadsheet Nebular Analysis Package
}

\author{
Curtis A.P. Krawchuk ${ }^{1}$, Marshall L. McCall ${ }^{1}$, Michael Komljenovic ${ }^{1}$, \\ Robin Kingsburgh ${ }^{1}$, Michael Richer ${ }^{2}$ and Christopher Stevenson ${ }^{3}$ \\ ${ }^{1}$ York Univ., Dept. of Physics and Astronomy, Toronto, Ontario, Canada; \\ ${ }^{2}$ DAEC, Obs. de Meudon, Meudon, France; \\ ${ }^{3}$ Memorial Univ., Dept. of Physics and Physical Oceanography, St. John's, Nlfd., Canada
}

A flexible and extensible software package for analyzing two-dimensional and multiobject spectra of nebulae has been implemented within Microsoft Excel (version 5). From lists of calibrated fluxes (and errors) for lines in a series of spectra, the program first identifies transitions by referring to wavelengths generated from atomic data. Colour excesses can be computed from a reddening law and ratio of total to selective extinction of the user's choice. Line fluxes can be corrected for stellar absorption and reddening and reported in a format suitable for publication. Temperatures, densities, ionic abundances, ionization correction factors, and total abundances can be computed. (The interface for the temperture/density calculation is shown below.) Emission coefficients for forbidden transitions are calculated using an adaptation of the FIVEL five-level atom routine (DeRobertis, 1987). As well, emission coefficients are supplied for an extensive set of recombination lines. The user has complete control over which spectral lines are used in an analysis - there are no pre-ordained methods. Abundance analyses can be performed using the physical conditions either measured or specified for each spectrum. Uncertainties are propagated throughout. The atomic data used in all of the calculations are easily updated or augmented by the user. Since the software is embedded in Microsoft Excel, graphical representations of the data are easily created, and extended analyses using the full functionality of the spreadsheet are easily implemented.

Email Contact: mccall@aries.phys.yorku.ca

REFERENCES

DeRobertis, M. M., Dufour, R. J., Hunt, R. W. 1987, JRASC, 81, 195. 